\title{
Torsional Vibration Suppression of A Gear System Installed with A Damping Ring
}

\author{
Yi-Long WANG ${ }^{1}$, Deng-Qing CAO ${ }^{1, a}$, Qing-Yang WANG ${ }^{2}$ and Yang YANG ${ }^{1}$
}

\author{
1 Harbin Institute of Technology, School of Astronautics, 150001 Harbin, China \\ 2Tsinghua university, School of Aerospace Engineering, 100084 Beijing, China
}

Keywords: Gear transmission, Damping ring, Stribeck friction model, Harmonic balance method, Vibration characteristic

\begin{abstract}
In this paper, the torsional vibration suppression is investigated for a gear system installed with a damping ring. The dynamic model of the gear-damping ring system is established, where the friction between the gear and the ring is described by the Stribeck model. The harmonic balance method is employed to work out the approximate analytical solution of the system. Meanwhile, a practical example is given to compare the analytical results with those from numerical simulation and to illustrate that the proposed analytical approach is valid. The amplitudefrequency characteristic of the system is then analysed and the resonant peak is found to be reduced with the increase of the friction.
\end{abstract}

\section{Introduction}

As is known in the field of mechanical transmission, the gear transmission system is widely used in power and motion transmission device in rotating machinery field due to the advantage of highpower range, high transmission efficiency, and long service life [1]. Since the noise in the gear transmission system is significant and vibration is a major source of the noise, it is very crucial to investigate the vibration of gear transmission systems [2].

The vibration of a gear transmission system includes torsional vibration, bending vibration and axial vibration. Because of the transmission error which arises from tooth profile error, periodic variation of the gear meshing stiffness, deformation of tooth during engagement as well as manufacturing errors, etc., the gear teeth are excited at the meshing point, causing the torsional vibration and consequently leading to the bending vibration and axial vibration as well [3-4], especially in spiral bevel gear [5]. There are some approaches to suppress vibration in mechanical transmission, such as vibration isolation, application of dynamic vibration absorber and vibration damping technology, etc., among which the damping ring technology is effectively applied to improve the vibration performance of gear transmission system.

In the aspect of research on gear transmission system, in 1981, Krezer [6] introduced a tool for analysing tooth contact and motion transmission errors of spiral bevel and hypoid gear sets in the loaded state. In their work, load, surface mismatches, tooth deformation and tooth bending were considered to calculate the position of contact spot on the tooth surface and the transmission error based on standard tooth contact analysis programs (TCA) and blank dimensions. According to the results of calculation in [6], the vibration performance of gear was obtained empirically. Kahraman and Singh [7] proposed a spur gear pair model with backlash and transmission error and solved the non-linear dynamic equations by harmonic balance method.

In the aspect of research on damping ring, Okamura and Suzuki [8] clarified the damping mechanism and the dynamic behaviour of the sound damping rings. In their work, the characteristics of damping rings and the effects resulting from transmission error on the vibration of gear were investigated by experiments. Wang et al. [3] established an axial vibration equations of a gear-damping ring system and proposed an approximate analytical solution with the harmonic balance method, and then discussed the amplitude-frequency characteristic based on the analytical solution. Firrone and Zucca [9] presented a method for the design of ring dampers for gears, which relied on the nonlinear calculation of the forced response of the frictionally damped gear in the 
frequency domain by means of the harmonic balance method. Later, Firrone and Zucca presented a passive approach based on the application of a ring damper to reduce the vibration level in [10], and a numerical method which coupled the static and dynamic equilibrium equations of the assembly were presented to be applied to a dummy bevel gear and to a ring damper having a flat punch contact area.

Most of researches on damping ring are mainly studied based on the empirical formula and a large amount of experiments, which may lead to large research cost and long production cycle. Thus, theoretical analysis of gear-damping ring system is particularly necessary. In this paper, a dynamic model for torsional vibration of a gear-damping ring system is established, where the friction between the gear and the ring is described by the Stribeck model. The harmonic balance method is employed to work out the approximate analytical solution of the system. Meanwhile, assuming that there is a certain damping material installed between the gear and the damping ring, which contributes to vibration suppression of the system, a practical example is given to compare the analytical results with those from numerical simulation and to illustrate that the proposed analytical approach is valid. After that, the amplitude-frequency characteristics of the system under different friction conditions are analysed. In addition, the influences of frictional parameters on the dynamic characteristic are discussed as well.

\section{Mathematical Model}

A simplified gear transmission system is shown in Figure 1. In order to investigate the influence of damping ring on the torsional vibration suppression of gear, a simplified mathematical dynamic model is set up to analyse the gear torsional vibration system with the application of damping ring. In this simplified model, it is assumed that the transmission shafts in this system are rigid, meaning that the bending and axial vibration is insignificant. Furthermore, since both the transmission error and time-varying mesh stiffness can be represented by Fourier series having dominant components with frequencies at integer multiples of the tooth passing frequency which is equal to the number of gear teeth times the shaft rotating speed [4], the external excitation subjected to the driven gear at meshing point is regarded as a periodical excitation in the form of $P_{0} \sin \omega t$.

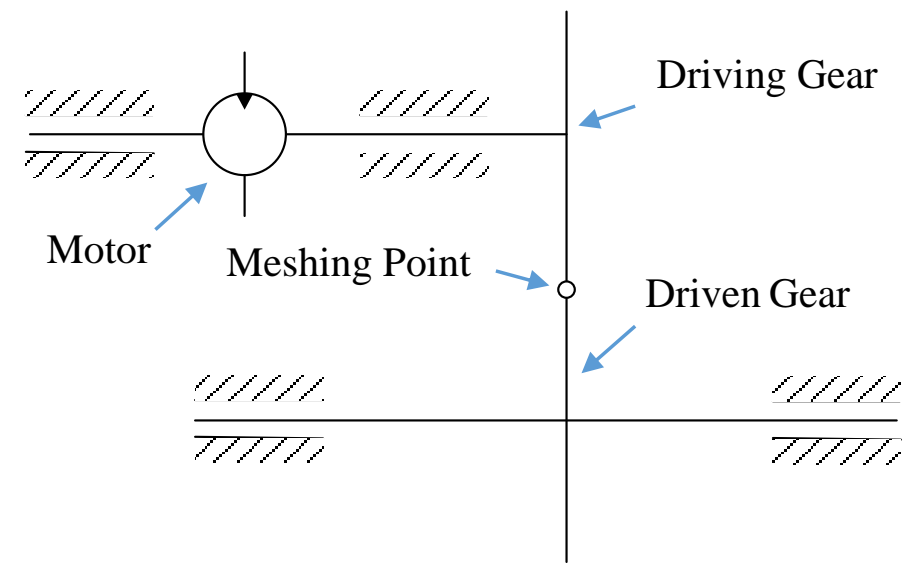

Figure 1. A simplified gear transmission system.

\section{Dynamic Equations}

To represent the torsional vibration of the gear-damping ring system, the two-degree-freedom model is set up, as shown in Figure 2.

Compared to the damping ring, all of the damping forces caused by the gear structure are insignificant and thus ignored in this paper. Furthermore, the damping ring and the gear structure comprising gear and bearing are all considered as concentrated masses. $m_{1}, J_{1}, k_{1}, r_{1}$ denote the equivalent mass, inertial moment, torsional stiffness and the radius of the gear, respectively. $m_{2}, J_{2}, r_{2}$ denote the equivalent, inertial moment, torsional stiffness and the radius of the damping 
ring, respectively. Damping coefficient of the damping ring and the sliding friction between the gear and the damping ring are denoted by $c_{2}$ and $f$ respectively. $\theta_{1}, \theta_{2}$ represent the angular displacement of the gear and the damping ring respectively. The gear is subjected to an external excitation $P_{0} \sin \omega t$.

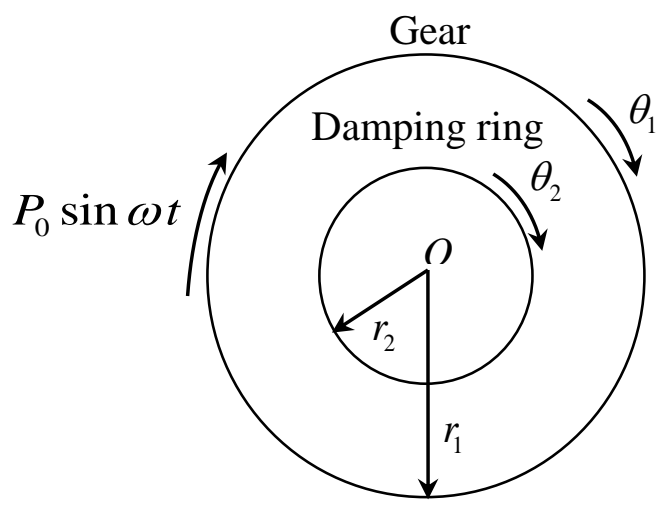

Figure 2. The gear-damping ring system model.

The Stribeck model instead of the classical Coulomb friction model is considered to establish the dynamic equations in this paper. This is because under steady conditions, the friction decreases with the increasing of relative velocity in the low relative velocity regime. This drooping characteristic of the friction force in the low relative velocity regime is known as the 'Stribeck effect' after the scientist Richard Stribeck for his work on this typical friction characteristics [11]. Therefore, in order to establish the accurate dynamic equations possibly, the 'Stribeck effect' need to be considered. $\mathrm{Li}$ and Pavelescu [12] presented an exponential function model which obviously described this nonlinear relationship between the dry friction coefficient and the relative velocity of the two contact blocks, which has been used by many researchers for different kinds of investigations. This function model is given by [12]:

$$
f=F \operatorname{sgn}\left(v_{r}\right)-D_{1} v_{r}+D_{2} v_{r}^{3} .
$$

According to the D 'Alembert principle, the dynamic equations of the system can be expressed as:

$$
\begin{gathered}
J_{1} \ddot{\theta}_{1}+k_{2}\left(\theta_{1}-\theta_{2}\right)+c_{2} r_{2}^{2}\left(\dot{\theta}_{1}-\dot{\theta}_{2}\right)+\operatorname{sgn}\left(\dot{\theta}_{1}-\dot{\theta}_{2}\right) F r_{2} \\
-D_{1}\left(\dot{\theta}_{1}-\dot{\theta}_{2}\right) r_{2}^{2}+D_{2}\left(\dot{\theta}_{1}-\dot{\theta}_{2}\right)^{3} r_{2}^{4}+k_{1} \theta_{1}=P_{0} r_{1} \sin \omega t . \\
J_{2} \ddot{\theta}_{2}-k_{2}\left(\theta_{1}-\theta_{2}\right)-c_{2} r_{2}^{2}\left(\dot{\theta}_{1}-\dot{\theta}_{2}\right)-\operatorname{sgn}\left(\dot{\theta}_{1}-\dot{\theta}_{2}\right) F r_{2}-D_{1}\left(\dot{\theta}_{1}-\dot{\theta}_{2}\right) r_{2}^{2}+D_{2}\left(\dot{\theta}_{1}-\dot{\theta}_{2}\right)^{3} r_{2}^{4}=0 .
\end{gathered}
$$

where $F$ is the sliding friction between damping ring and gear, and is nearly equal to the maximum static friction.

\section{Analytical Solution of Torsional Vibration}

According to the results in the Paper [3], the Coulomb friction model can be represented by a Fourier series. Consequently, the harmonic balance method can be employed to solve the dynamic equations analytically.

Firstly, let $\varphi=x_{1}-x_{2}$, then $\dot{\varphi}=\dot{x}_{1}-\dot{x}_{2}, \ddot{\varphi}=\ddot{x}_{1}-\ddot{x}_{2}$ can be obtained. The $(\bullet)$ notation represents the derivative with respect to time. By substituting $\varphi, \dot{\varphi}$ and $\ddot{\varphi}$ into Eq. (1) and (2), the dynamic equations can be rewritten as:

$$
\begin{aligned}
& J_{1} \ddot{\theta}_{1}+k_{1} \theta_{1}+k_{2} \varphi+c_{2} r_{2}^{2} \dot{\varphi}+\operatorname{sgn}(\dot{\varphi}) F r_{2}-D_{1} \dot{\varphi} r_{2}^{2}+D_{2} \dot{\varphi}^{3} r_{2}^{4}=P_{0} r_{1} \sin \omega t \\
& J_{2} \ddot{\theta}_{1}-J_{2} \ddot{\varphi}-k_{2} \varphi-c_{2} r_{2}^{2} \dot{\varphi}-\operatorname{sgn}(\dot{\varphi}) F r_{2}+D_{1} \dot{\varphi} r_{2}^{2}-D_{2} \dot{\varphi}^{3} r_{2}^{4}=0 .
\end{aligned}
$$


For a forced vibration system, the dynamic responses include transient and stationary parts. In order to investigate the influence of the damping ring on the gear, stationary responses are picked out. Herein, only fundamental harmonic terms are considered [3], and it is assumed that the initial formulation has the following forms

$$
\theta_{1}=A_{1} \sin \left(\omega t-\alpha_{1}\right) \quad \varphi=A_{2} \sin \left(\omega t-\alpha_{2}\right) .
$$

Thus $\dot{\varphi}=A_{2} \omega \cos \left(\omega t-\alpha_{2}\right)$, and the fundamental harmonic term of $F \operatorname{sgn}\left(v_{r}\right)$ in Eq. (1) can be expressed in the following form

$$
\tilde{f}=\operatorname{sgn}\left(A_{2} \omega \cos \theta\right) F=\frac{4}{\pi} \cos \left(\omega t-\alpha_{2}\right) F .
$$

Then, Eqs. (4) and (5) can be simplified to the form:

$$
\left\{\begin{array}{l}
\left(k_{1}-J_{1} \omega^{2}\right) A_{1} \sin \left(\omega t-\alpha_{1}\right)+\left[\left(c_{2}-D_{1}\right) r_{2}^{2} A_{2} \omega+\frac{4 F r_{2}}{\pi}\right] \cos \left(\omega t-\alpha_{2}\right) \\
+k_{2} A_{2} \sin \left(\omega t-\alpha_{2}\right)+\frac{1}{4} D_{2} r_{2}^{4} A_{2}^{3} \omega^{3}\left[3 \cos \left(\omega t-\alpha_{2}\right)+\cos 3\left(\omega t-\alpha_{2}\right)\right]=P_{0} r_{1} \sin \omega t \\
-J_{2} \omega^{2} A_{1} \sin \left(\omega t-\alpha_{1}\right)-\left[\left(c_{2}-D_{1}\right) r_{2}^{2} A_{2} \omega+\frac{4 F r_{2}}{\pi}\right] \cos \left(\omega t-\alpha_{2}\right) \\
-\left(k_{2}-J_{2} \omega^{2}\right) A_{2} \sin \left(\omega t-\alpha_{2}\right)-\frac{1}{4} D_{2} r_{2}^{4} A_{2}^{3} \omega^{3}\left[3 \cos \left(\omega t-\alpha_{2}\right)+\cos 3\left(\omega t-\alpha_{2}\right)\right]=0 .
\end{array}\right.
$$

Neglecting the terms containing $\sin 3 \omega t$ and $\cos 3 \omega t$ in Eq. (8), we have [3]

$$
\left\{\begin{array}{l}
{\left[\left(k_{1}-J_{1} \omega^{2}\right) A_{1} \cos \alpha_{1}+k_{2} A_{2} \cos \alpha_{2}+B A_{2} \sin \alpha_{2}\right] \sin \omega t} \\
+\left[-\left(k_{1}-J_{1} \omega^{2}\right) A_{1} \sin \alpha_{1}-k_{2} A_{2} \sin \alpha_{2}+B A_{2} \cos \alpha_{2}\right] \cos \omega t=P_{0} r_{1} \sin \omega t, \\
{\left[-J_{2} \omega^{2} A_{1} \cos \alpha_{1}-\left(k_{2}-J_{2} \omega^{2}\right) A_{2} \cos \alpha_{2}-B A_{2} \sin \alpha_{2}\right] \sin \omega t} \\
+\left[J_{2} \omega^{2} A_{1} \sin \alpha_{1}+\left(k_{2}-J_{2} \omega^{2}\right) A_{2} \sin \alpha_{2}-B A_{2} \cos \alpha_{2}\right] \cos \omega t=0 .
\end{array}\right.
$$

where $B=\left(c_{2}-D_{1}\right) r_{2}^{2} \omega+\frac{4 F r_{2}}{\pi A_{2}}+\frac{3}{4} D_{2} r_{2}^{4} A_{2}^{2} \omega^{3}$.

Let $a=A_{1} \sin \alpha_{1}, b=A_{1} \cos \alpha_{1}, c=A_{2} \sin \alpha_{2}, d=A_{2} \cos \alpha_{2}$. Comparing both sides of Eq. (9) using the harmonic balance method yields

$$
\left\{\begin{array}{l}
\left(k_{1}-J_{1} \omega^{2}\right) b+k_{2} d+B c=P_{0} r_{1}, \\
\left(-k_{1}+J_{1} \omega^{2}\right) a-k_{2} c+B d=0, \\
\left(-k_{2}+J_{2} \omega^{2}\right) d-J_{2} \omega^{2} b-B c=0, \\
\left(k_{2}-J_{2} \omega^{2}\right) c+J_{2} \omega^{2} a-B d=0 .
\end{array}\right.
$$

Eq. (10) are linear equations of $a, b, c, d$. By simplifying Eq. (10), $a, b$ can be expressed as

$$
a=\frac{J_{2} \omega^{2} c}{-k_{1}+J_{1} \omega^{2}+J_{2} \omega^{2}}, \quad b=\frac{P_{0} r_{1}-J_{2} \omega^{2} d}{k_{1}-J_{1} \omega^{2}-J_{2} \omega^{2}} .
$$

By substituting Eq. (11) into Eq. (10), the following linear equations are obtained:

$$
M d+N c=-J_{2} \omega^{2} P_{0} r_{1}, M c-N d=0
$$


where $\left\{\begin{array}{l}M=\left(k_{1}-\omega^{2} J_{1}\right)\left(k_{2}-\omega^{2} J_{2}\right)-\omega^{2} J_{2} k_{2}, \\ N=B\left(k_{1}-J_{1} \omega^{2}-J_{2} \omega^{2}\right)\end{array}\right.$

The expressions of $c, d$ can be obtained by solving Eq. (12). Then, by substituting the expressions of c, d, the solutions of Eq. (11) can also be obtained. Therefore, the solution of the Eq. (10) is

$$
\left\{\begin{array}{l}
a=\frac{J_{2}^{2} \omega^{4} B P_{0} r_{1}}{M^{2}+N^{2}}, \quad b=\frac{\left(M^{2}+J_{2}^{2} \omega^{4} M+N^{2}\right) B P_{0} r_{1}}{N\left(M^{2}+N^{2}\right)}, \\
c=\frac{-J_{2} \omega^{2} P_{0} r_{1} N}{M^{2}+N^{2}}, \quad d=\frac{-J_{2} \omega^{2} P_{0} r_{1} M}{M^{2}+N^{2}} .
\end{array}\right.
$$

Because the expression of B contains $A_{2}$, Eq. (13) is implicit. Hence, it is necessary to obtain a further solution.

Let $D=k_{1}-J_{1} \omega^{2}-J_{2} \omega^{2}$. Then

$$
\left\{\begin{array}{l}
A_{1}=\sqrt{a^{2}+b^{2}}=\sqrt{\frac{\left(M+J_{2}^{2} \omega^{4}\right)^{2}+N^{2}}{M^{2}+N^{2}}} \frac{P_{0} r_{1}}{|D|}, \\
A_{2}=\sqrt{c^{2}+d^{2}}=\frac{J_{2} \omega^{2} P_{0} r_{1}}{\sqrt{M^{2}+N^{2}}} .
\end{array}\right.
$$

and

$$
\left\{\begin{array}{l}
\alpha_{1}=\arctan \left(\frac{a}{b}\right)=\arctan \left(\frac{J_{2}^{2} \omega^{4} N}{M^{2}+J_{2}^{2} \omega^{4} M+N^{2}}\right) \\
\alpha_{2}=\arctan \left(\frac{c}{d}\right)=\frac{N}{M}
\end{array}\right.
$$
that

Together with the expression of $N$, Eq. (14) can be further derived by squaring the both sides, so

$$
M^{2} A_{2}^{2}+\left[\left(c_{2}-D_{1}\right) A_{2} r_{2}^{2} \omega+\frac{4 F r_{2}}{\pi A_{2}}+\frac{3}{4} D_{2} r_{2}^{4} A_{2}^{2} \omega^{3}\right]^{2} D^{2} A_{2}^{2}=J_{2}^{2} \omega^{4} P_{0}^{2} r_{1}^{2}
$$

After further simplification, an algebraic equation of sixth degree is obtained:

$$
\begin{aligned}
& \frac{9}{16} D_{2}^{2} r_{2}^{8} D^{2} \omega^{6} A_{2}^{6}+\frac{3}{2} D_{2} r_{2}^{6} D^{2}\left(c_{2}-D_{1}\right) \omega^{4} A_{2}^{4}+\frac{6 F}{\pi} D_{2} r_{2}^{5} D^{2} \omega^{3} A_{2}^{3}-J_{2}^{2} \omega^{4} P_{0}^{2} r_{1}^{2} \\
& +\left[M^{2}+\left(c_{2}-D_{1}\right)^{2} r_{2}^{4} D^{2} \omega^{2}\right] A_{2}^{2}+\frac{8 F\left(c_{2}-D_{1}\right) r_{2}^{3} D^{2} \omega}{\pi} A_{2}+\frac{16 F^{2} r_{2}^{2} D^{2}}{\pi^{2}}=0 .
\end{aligned}
$$

Obviously, there are six roots in Eq. (17). Taking into account the actual physical characteristic, the root should be positive and real. Then, the vibration amplitude $A_{1}$ of the gear and the phases $\alpha_{1}, \alpha_{2}$ can be determined by computing $A_{2}$. Finally, the response of the gear-damping ring system can be determined.

\section{Numerical Simulation and Results}

The Stribeck friction model [15] is given as 


$$
\left\{\begin{array}{l}
\mu\left(v_{r}\right)=\mu_{s} \operatorname{sgn}\left(v_{r}\right)-\kappa_{1} v_{r}+\kappa_{3} v_{r}^{3}, \\
\kappa_{1}=(3 / 2)\left(\left|\mu_{s}\right|-\left|\mu_{m}\right|\right) /\left|v_{m}\right|, \\
\kappa_{3}=(1 / 2)\left(\left|\mu_{s}\right|-\left|\mu_{m}\right|\right) /\left|v_{m}\right|^{3} .
\end{array}\right.
$$

where $\mu_{s}$ is the coefficient of static friction, $v_{m}$ is the relative linear velocity between the gear and the damping ring which is a coefficient corresponding to the minimum coefficient $\mu_{m}$ of kinetic friction.

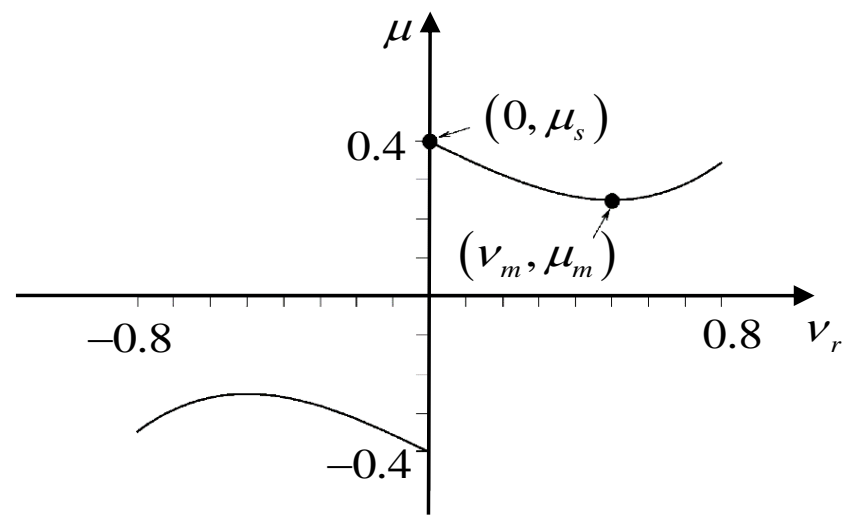

Figure 3. Coefficient of Friction as given by Eq. (18).

There is a relationship between the coefficients as $\mu_{m} \leq \mu_{s}$, and $\kappa_{1}, \kappa_{2} \geq 0$ [13]. Herein, the simulation parameters are set as $\mu_{s}=0.4, \mu_{m}=0.25, v_{m}=0.5 \mathrm{~m} / \mathrm{s}$ according to an experimental prototype. The diagram of $\mu$ is shown in Figure 3.

Denote $N$ as the pressure between gear and damping ring, and the friction can be calculated by Eqs. (1), (18) and the expression:

$$
D_{1}=\kappa_{1} F_{N}, \quad D_{2}=\kappa_{3} F_{N}, \quad F=\mu_{s} F_{N}
$$

The pressure $F_{N}$ is set as $2.5 \mathrm{~N}$ in this simulation study, then $F=1 \mathrm{~N}, D_{1}=1.125, D_{2}=1.5$ can be obtained from Eq. (19). The parameters of the system are given as shown in Table 1.

Table 1. The parameters of the gear-damping ring system.

\begin{tabular}{|l|l|}
\hline Item & Description \\
\hline Radius of the gear $r_{1}$ & $0.5 \mathrm{~m}$ \\
\hline Torsional stiffness of the gear $k_{1}$ & $10000 \mathrm{~N} \cdot \mathrm{m} / \mathrm{rad}$ \\
\hline Inertial moment of the gear $J_{1}$ & $1 \mathrm{~kg} \times \mathrm{m}^{2}$ \\
\hline Radius of the damping ring $r_{2}$ & $0.2 \mathrm{~m}$ \\
\hline Torsional stiffness of the damping ring $k_{2}$ & $1000 \mathrm{~N} \cdot \mathrm{m} / \mathrm{rad}$ \\
\hline Inertial moment of the damping ring $J_{2}$ & $0.1 \mathrm{~kg} \times \mathrm{m}^{2}$ \\
\hline Damping coefficient of the damping ring $c_{2}$ & $50 \mathrm{~N} \cdot \mathrm{s} / \mathrm{m}$ \\
\hline Maximum external excitation $P_{0}$ & $30 \mathrm{~N}$ \\
\hline The frequency of external excitation $\omega$ & $85.85 \mathrm{rad} / \mathrm{s}$ \\
\hline
\end{tabular}

With the parameters given in Table 1, it is calculated that the first two natural frequency of the linearized system are $\omega_{1}=85.85 \mathrm{rad} / \mathrm{s}$ and $\omega_{2}=116.30 \mathrm{rad} / \mathrm{s}$, respectively. 
By substituting the parameters into Eqs. (14) and (15) we have $A_{1}=0.014073 \mathrm{rad}$ and $\alpha_{1}=1.113768 \mathrm{rad}$. The stationary response curve of analytical solution under the excitation is shown in Figure 4. The analytical stationary response can be expressed as

$$
\theta_{1}=0.014073 \sin (85.85 t+1.113768)
$$

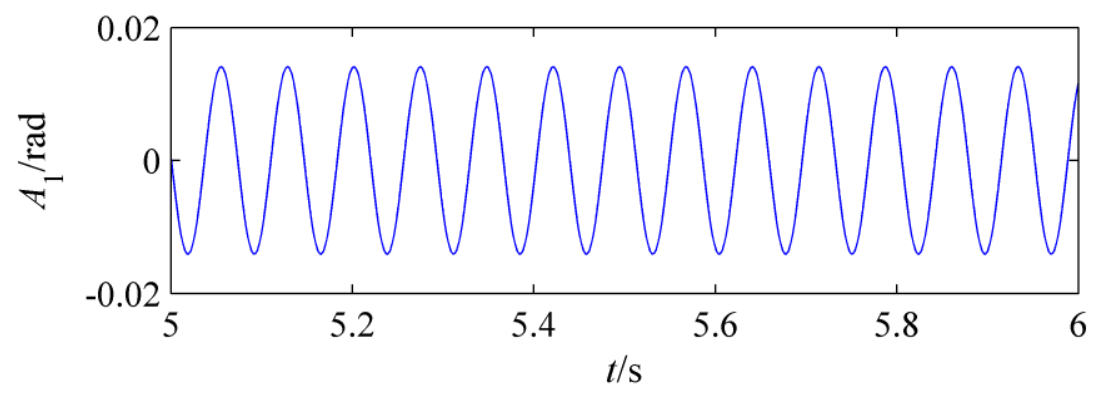

Figure 4 . The stationary response curve of analytical solution.

For comparative analysis, the stationary response curve of numerical solution when $F_{N}=2.5 \mathrm{~N}$ is given in Figure 5, where the amplitude $A_{2}$ is $0.014065 \mathrm{rad}$. It is also observed that the stationary response curves of both solutions are basically consistent, revealing that the approximate analytical solution is valid.

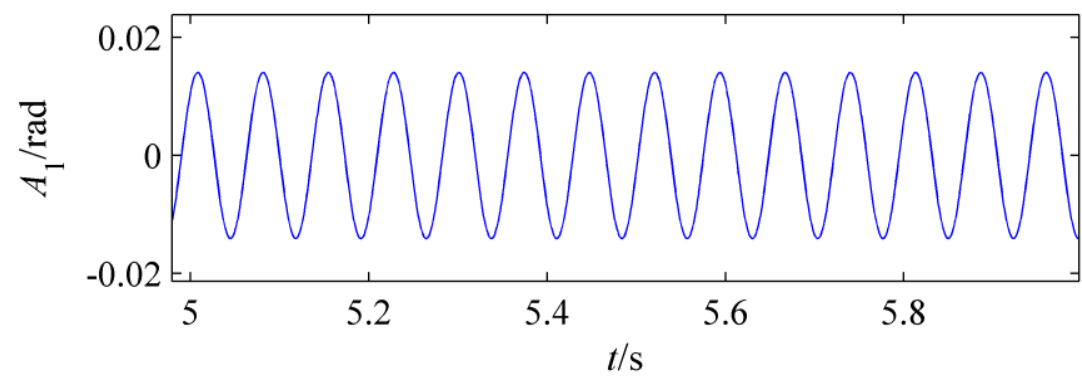

Figure 5. The stationary response curve of numerical solution.

For further verification, the comparison of both solutions of this system when the pressure $F_{N}$ is $2.5 \mathrm{~N}, 5 \mathrm{~N}, 7.5 \mathrm{~N}, 10 \mathrm{~N}$ are shown in Table 2 , respectively. It is obvious that the relative errors between both solutions are quite small. Thus, the approximate analytical solution is as accurate as numerical solution.

Table 2. Comparison of both solutions of this system under different pressures.

\begin{tabular}{|l|l|l|l|l|}
\hline Pressure $F_{N}(\mathrm{~N})$ & 2.5 & 5 & 7.5 & 10 \\
\hline Analytical solution & 0.014073 & 0.013791 & 0.013510 & 0.013228 \\
\hline numerical solution & 0.014065 & 0.013782 & 0.013490 & 0.013201 \\
\hline Error $(\%)$ & -0.057 & -0.065 & -0.148 & -0.206 \\
\hline
\end{tabular}

According to Eqs. (14) and (19), the amplitude-frequency characteristic curves of the system are plotted in Figure 6 for the pressure $F_{N}=0 \mathrm{~N}, 10 \mathrm{~N}, 20 \mathrm{~N}$, respectively. The resonant amplitude and frequency values are listed in the Table 3.

It can be observed from Fig. 6 that the resonant peak reduces with the increase of the pressure $F_{N}$, which means that the increase of friction is indeed helpful to vibration suppression and noise reduction. The friction between the gear and the ring depends on the friction coefficient and the pressure $F_{N}$, while $F_{N}$ varies with the compression of the damping ring. To sum up, for better 
suppression of vibration, the material and compression of the damping ring are necessary to be considered in the design.

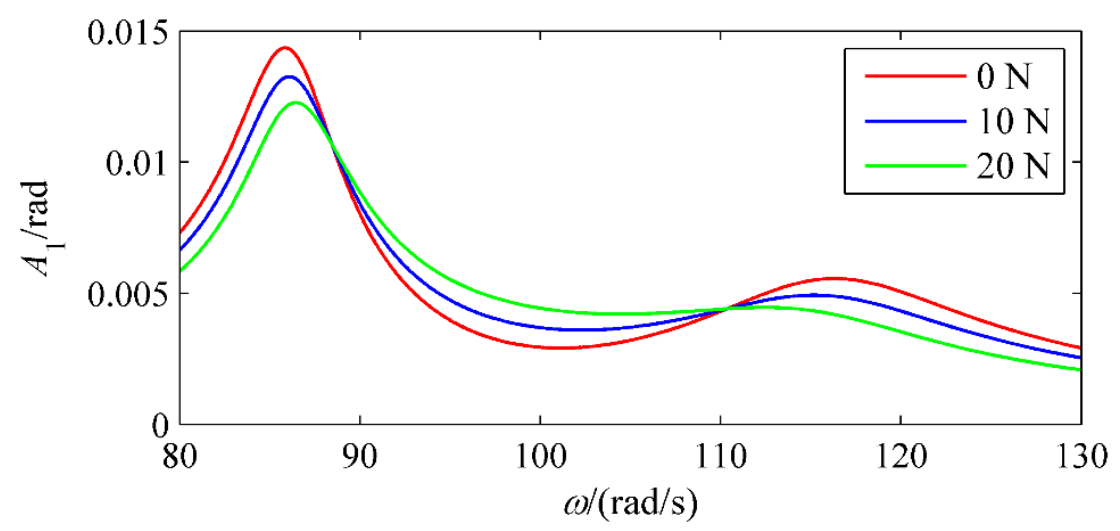

Figure 6 . The amplitude-frequency characteristic curve

Table 3. The resonant amplitude-frequency values.

\begin{tabular}{|c|c|c|c|c|}
\hline Pressure $F_{N}(\mathrm{~N})$ & $\begin{array}{l}1^{\text {st }} \text { resonant } \\
\text { frequency }(\mathrm{Hz})\end{array}$ & $\begin{array}{l}1^{\text {st }} \text { resonant } \\
\text { amplitude }(\mathrm{rad})\end{array}$ & $\begin{array}{l}2^{\text {nd }} \text { resonant } \\
\text { frequency }(\mathrm{Hz})\end{array}$ & $\begin{array}{l}2^{\text {nd }} \text { resonant } \\
\text { amplitude }(\mathrm{rad})\end{array}$ \\
\hline 0 & 85.85 & 0.014356 & 116.30 & 0.005558 \\
\hline 10 & 86.10 & 0.013260 & 115.20 & 0.004927 \\
\hline 20 & 86.45 & 0.012269 & 112.40 & 0.004460 \\
\hline
\end{tabular}

As noticed in Table 3, due to the nonlinear characteristics of the system, the resonant frequencies are different from the linear system. As seen, the 1st resonant frequencies are gently lower than the natural frequency of the corresponding linear system and rise with the increase of the pressure $c_{1}$. Conversely, the 2nd resonant frequencies are little higher and decrease with the increase of the pressure $F_{N}$.

For investigation of relationship among the amplitude $A_{1}$, damping coefficient of the damping ring $c_{2}$, frequency of external excitation $\omega$, Eqs. (14) and (17) are used with the pressure $F_{N}=20 \mathrm{~N}$ in this simulation. As shown in Figure 7, the relationship among the three parameters is presented. The 2 nd resonant is ignored because $A_{1}$ is insignificant in this case. It is also observed that the $1 \mathrm{st}$ resonant $A_{1}$ decreases before $c_{2}$ reaches a certain value (around $87 \mathrm{~N} \cdot \mathrm{s} / \mathrm{m}$ ) and increases later, meaning that there is an optimal value of $c_{2}$ for vibration suppression of this system. Furthermore, it is noted that the 1st resonant frequency goes up with the increase of $c_{2}$. This is because the varying damping coefficient of the damping ring $c_{2}$ affects the characteristic of this system due to the nonlinear friction $f$.

Similarly, for investigation of the relationship among $A_{1}, F_{N}$ and $\omega$, the damping coefficient $c_{2}$ is set to be $90 \mathrm{Ns} / \mathrm{m}$ which is near the optimal value of $c_{2}$. As shown in Figure 8 , in addition to insignificant resonant frequency shift, the slight change of the 1 st resonant $A_{1}$ with the increase of $F_{N}$ reveals that $c_{2}$ has a dominant effect on the 1 st resonant $A_{1}$ instead of $F_{N}$ in this case. Meanwhile, it is observed that the increase of $F_{N}$ leads to a similar trend of the 1 st resonant $A_{1}$ as $c_{2}$ does, revealing that there is also an optimal value of $F_{N}$ for vibration suppression of this system. Furthermore, compared to this case, when $c_{2}=50 \mathrm{~N} \cdot \mathrm{s} / \mathrm{m}, F_{N}$ has a dominant effect on the resonant peaks. Thus, only when $c_{2}$ is designed to be small, by adjusting $F_{N}$ may lead to a better vibration suppression of the resonant peak.

The reason why $F_{N}$ and $c_{2}$ have a similar effect on the 1 st resonant $A_{1}$ is that both parameters are related to the relative motion between damping ring and gear. If $F_{N}$ and $c_{2}$ are too small, even though the relative motion is significant, the vibration energy reduces slowly. And if $F_{N}$ and $c_{2}$ are too large, the suppression of vibration energy is also insignificant due to the slight relative motion. 


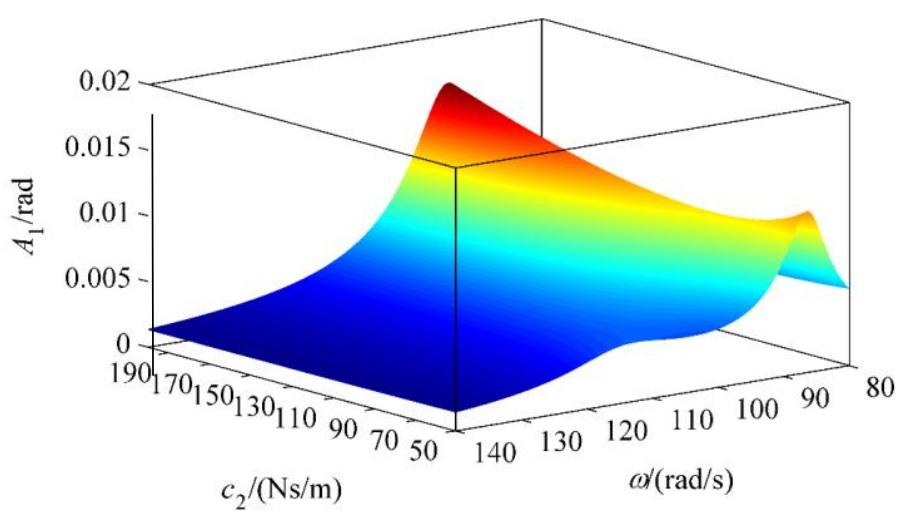

Figure 7. The surface plot of the relationship among $A_{1}, c_{2}$ and $\omega$.

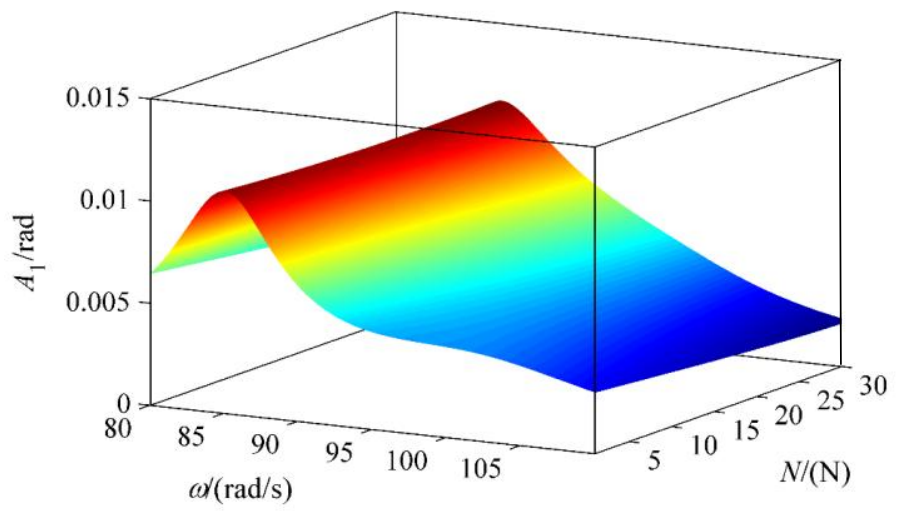

Figure 8. The surface plot of the relationship among $A_{1}, F_{N}$ and $\omega$.

As shown in Figure 8, in addition to insignificant resonant frequency shift, the slight change of the 1 st resonant $A_{1}$ with the increase of $F_{N}$ reveals that $c_{2}$ has a dominant effect on the 1 st resonant $A_{1}$ instead of $F_{N}$ in this case. Meanwhile, it is observed that the increase of $F_{N}$ leads to a similar trend of the 1 st resonant $A_{1}$ as $c_{2}$ does, revealing that there is also an optimal value of $F_{N}$ for vibration suppression of this system. Furthermore, compared to this case, when $c_{2}=50 \mathrm{~N} \cdot \mathrm{s} / \mathrm{m}, F_{N}$ has a dominant effect on the resonant peaks. Thus, only when $c_{2}$ is designed to be small, by adjusting $F_{N}$ may lead to a better vibration suppression of the resonant peak.

The reason why $F_{N}$ and $c_{2}$ have a similar effect on the 1 st resonant $A_{1}$ is that both parameters are related to the relative motion between damping ring and gear. If $F_{N}$ and $c_{2}$ are too small, even though the relative motion is significant, the vibration energy reduces slowly. And if $F_{N}$ and $c_{2}$ are too large, the suppression of vibration energy is also insignificant due to the slight relative motion.

\section{Conclusion}

The suppression of torsion vibration of a gear system has been studied by installing a damping ring in the gear. The harmonic balance method was employed to work out the approximate analytical solution of the system and the comparison of the analytical results and the numerical results were performed. The amplitude-frequency curve of the system under different friction conditions were analysed. The following conclusions can be obtained:

(1) By comparing the analytical results with numerical results, the analytical approach has been shown to be valid.

(2) The resonant peak reduces significantly with the increase of the pressure $F_{N}$, especially when damping coefficient $c_{2}$ is small. 
(3) The damping coefficient $c_{2}$ and the pressure $F_{N}$ of the system have a similar effect on $A_{1}$, especially at the 1 st resonant peak. However, when $c_{2}$ is large enough, the effect on $A_{1}$ is dominated by $c_{2}$ while $F_{N}$ only has a slight effect.

(4) Due to the influence of the nonlinear friction, the varying of both the pressure $F_{N}$ and the damping coefficient $c_{2}$ may lead to resonant frequency shift, and the influence of $c_{2}$ on resonant frequency shift is more significant than that of $F_{N}$.

The results of this paper can be used as the theoretical basis for the design of gears applied in aero-engine and the guidance for further research.

\section{References}

1. C. F. Li, S. H. Zhou, J. Liu. Numerical simulation of a bending-torsion coupling gear transmission system. Appl. Mech. Mater., 448-453, 3403-3407. (2013)

2. J. D. Smith. Gear noise and vibration. (CRC Press, 1999)

3. Q. Y. Wang, D. Q. Cao, J. B. Yang. Axial vibration reduction characteristics of a gear system with a damping ring. J. Vib. Shock, 32(6), 190-194. (2013)

4. S. T. Choi, S. Y. Mau, S. T. Choi, S. Y. Mau. Dynamic analysis of geared rotor-bearing systems by the transfer matrix method. J. Mech. Design, 123(4), 562-568. (2001)

5. N. Peng, R. P. Zhu, H. Y. Bao, F. X. Lu. Study on the influence of type C damping ring structure parameter on dynamics characteristic of bevel gear transmission.J. Mech. Transm., 38(8), $1-5,17 .(2014)$

6. T. J. Krenzer. Tooth contact analysis of spiral bevel and hypoid gears under load. Earthmoving Industry Conference, Peoria, IL, United states, April 6-8, 1981. (2008)

7. A. Kahraman, R. Singh. Interactions between time-varying mesh stiffness and clearance nonlinearities in a geared system. J. Sound Vib., 146(1), 135-156. (1991)

8. H. Okamura, Y. Suzuki. An experimental study of sound damping rings for gears-dynamical behaviour and optimum design parameters for sound damping rings. In ASME. (1975)

9. C. M. Firrone, S. Zucca. A method for the design of ring dampers for gears in aeronautical applications. ASME 2010 International Mechanical Engineering Congress and Exposition, 134, 117-127. (2010)

10. C. M. Firrone, S. Zucca. Passive control of vibration of thin-walled gears: advanced modelling of ring dampers. Nonlinear Dynam., 76(1), 263-280. (2014)

11. A. Saha, M. Wiercigroch, K. Jankowski, P. Wahi, A. Stefański. Investigation of two different friction models from the perspective of friction-induced vibrations. Tribol. Int, 90(8), 185-197. (2015)

12. C. B. Li, D. Pavelescu. The friction-speed relation and its influence on the critical velocity of stick-slip motion. Wear, 82(3), 277-289. (1982)

13. J. J. Thomsen, A. Fidlin. Analytical approximations for stick-slip vibration amplitudes. Int. J. Nonlinear Mech., 38(3), 389-403. (2003) 